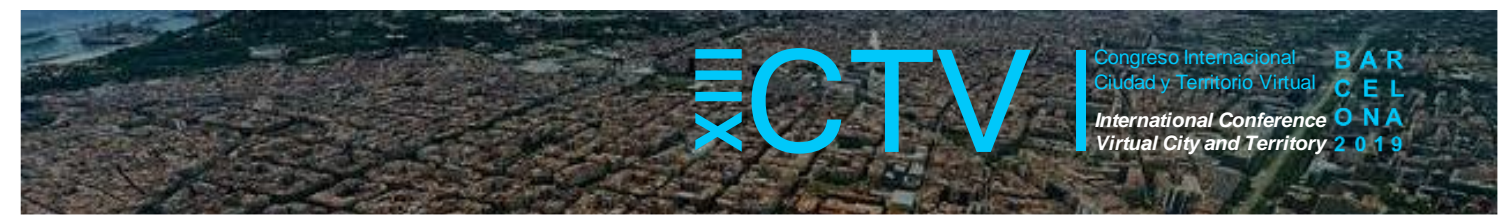

\title{
LA VERTICALIDAD EN LA VIVIENDA TURíSTICA. EL PROCESO DE TRANSFORMACIÓN DE PUERTO VALLARTA, JALISCO, MÉXICO
}

\author{
Bustos, David ${ }^{1 *}$; Chavoya, Jorge ${ }^{2}$ \\ Remisión inicial: 2019-07-31; Remisión definitiva: 2019-10-20; Publicación: 2019-12-21
}

Citación: Bustos, D. y Chavoya, J. (2019). La Verticalidad en la vivienda turística. El proceso de transformación de Puerto Vallarta, Jalisco, México. En XIII CTV 2019 Proceedings: XIII International Conference on Virtual City and Territory: "Challenges and paradigms of the contemporary city": UPC, Barcelona, October 2-4, 2019. Barcelona: CPSV, 2019, p. 8763. E-ISSN 2604-6512. DOI http://dx.doi.org/10.5821/ctv.8763

\section{Resumen}

Debido a la relevancia turística de ciudad de Puerto Vallarta, Jalisco, México, el crecimiento urbano, ha propiciado un flujo migratorio intenso que surge dando alojamiento como un proceso direccionado a la verticalidad en la vivienda turística, mirando atributos arquitectónicos en la planeación urbana de la ciudad. En estos nuevos esquemas la vivienda turística juega un papel determinante en la reconfiguración territorial de la ciudad, toda vez que detona la tendencia a la verticalización promoviendo a su vez nuevos atributos arquitectónicos con la modificación del paisaje urbano, todo esto inserto hasta el momento en un modelo extractivo. Es por ello que en esta investigación me he planteado las siguientes preguntas: ¿Qué factores urbanísticos y arquitectónicos determinan la opción de la verticalidad en la vivienda turística en la ciudad de Puerto Vallarta, Jalisco? y ¿Qué procesos urbanos propicia la vivienda turística y como responde la tendencia a la verticalización en la ciudad de Puerto Vallarta, Jalisco?, con el objetivo de identificar y demostrar los factores arquitectónicos y urbanísticos que han propiciado la transformación urbana y que han influido en la tendencia de la verticalidad en la vivienda turística en el municipio de Puerto Vallarta, Jalisco e identificar los nuevos atributos arquitectónicos, conceptualizar el concepto de la densificación en su expresión de verticalidad en la vivienda turística e identificar las transformaciones urbanas en este proceso. Para conseguir esta metodología es a través del método analítico de tipo cualitativo, mediante la técnica de la observación participante en los lugares de interés previamente seleccionados.

\section{Abstract}

Due to its popularity as a tourist destination, the city of Puerto Vallarta, Jalisco, Mexico is experiencing an intense urban growth, primarily as a migratory flow of new residents, that manifests itself through the appearance of new buildings that consist, primarily, of vertical tourist housing, according to the architectural terminology used in the urban-planning of the city. In these new schemes, tourist housing plays a decisive role in the territorial reconfiguration of the city. It triggers the tendency to verticalization which, in turn, promotes new architectural attributes that change the urban landscape. All this has been done, so far, in an extractive mode. That is why in this research I have asked myself the following questions: what urban-planning and architectural factors should impact the option of verticality in tourist housing in the city of Puerto Vallarta, Jalisco; and what urban-planning processes are affected by tourist housing, and what is the response to the trend towards verticalization in the city of Puerto Vallarta, Jalisco?, the objective is to identify; and demonstrate the architectural and urban factors that have led to the urban transformation, and have influenced the trend towards verticality in tourist housing in the municipality of Puerto Vallarta, Jalisco and to identify; the new architectural attributes, to develop the concept of densification as expressed through verticality in tourist housing, and to identify; recent and current urban transformations as a result of this process. This methodology is being completed using the qualitative analytical method, by means of the technique of participant observation in the previously selected places of interest.

Palabras Clave: Verticalidad; redensificación; vivienda turística; ciudad compacta

Key words: Verticality; redensification; tourist housing; compact city 1'Estudiante de la Maestría en Ciencias para el Desarrollo, la Sustentabilidad y el Turismo. Universidad de Guadalajara,
Centro Universitario de la Costa, Puerto Vallarta, Jalisco, México, https://orcid.org/0000-0002-3374-3359; ${ }^{2}$ Profesor
titular "A" Universidad de Guadalajara, Centro Universitario de la Costa. Líder del cuerpo académico UDG-CA-303,
Estudios de la Ciudad, Arquitectura. Puerto Vallarta, Jalisco, México. "Correo de contacto:
arq.david.bustos@hotmail.com 


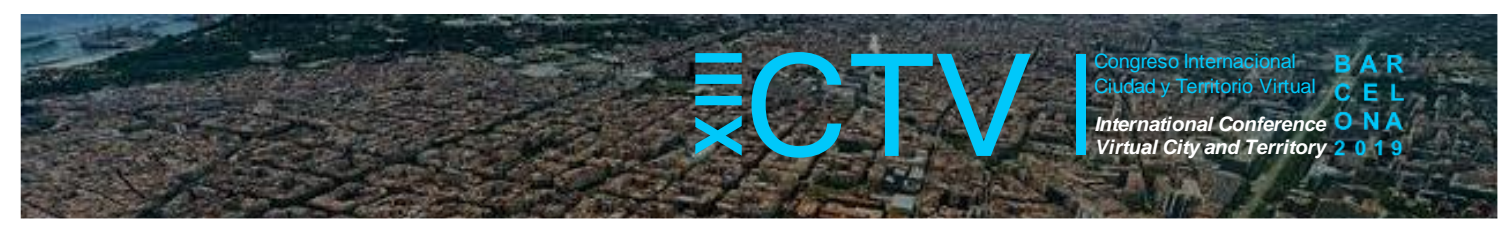

\section{Introducción}

El proceso de transformación de Puerto Vallarta, Jalisco, México, la verticalidad en la vivienda turística. "En un modelo densificador de metrópoli, es indispensable la creatividad en todos los aspectos: arquitectónicos, planificadores, políticos y sociales. La densificación en su expresión vertical constituye un motor para la innovación" (Chavoya, García, \& Rendón, 2009). Debido a la relevancia turística de ciudad de Puerto Vallarta, Jalisco, México, el crecimiento urbano, ha propiciado un flujo migratorio intenso que surge dando alojamiento como un proceso direccionado a la verticalidad en la vivienda turística, mirando atributos arquitectónicos en la planeación urbana de la ciudad.

En los últimos años Puerto Vallarta ha aumentado hasta el año del 2015 con una población de 275640 habitantes según datos de censos de población (INEGI, 2015), debido a sus oportunidades de desarrollo y quienes han decido habitar y quedarse en el ámbito comercial. El turismo es la principal fuente de ingreso, pero es por eso que en diferentes sectores "colonias" de la ciudad presenta un crecimiento acelerado crece caótica y mal ordenada o simplemente; edificación de construcciones mal planeadas para el crecimiento de la ciudad.

El turismo ha sido el factor el cual ha influido en el crecimiento de la ciudad de Puerto Vallarta, el más importante en el sector inmobiliario quien ha tomado posicionamiento en el hábitat de la población, en este caso más a extranjeros, quienes han elegido a Puerto Vallarta como un destino para vivir, por ser una ciudad tranquila y con atribuciones específicas, por tal motivo deciden radicar o comprar una propiedad o bien común en la ciudad. Hay algunos efectos positivos en ello, pero en general el rápido crecimiento demográfico. "Existe consenso respecto a las desventajas generadas por una expansión urbana incontrolada. Está demostrado en numerosos sectores que la falta de planeación del crecimiento produce efectos desastrosos en la ciudad: falta y/o es organización del transporte colectivo en distintas áreas de las ciudades, en general las de urbanización reciente; servicios públicos insuficientes e ineficientes (ausencia de infraestructura de agua, recolección de basura desigual en el territorio, etc.); equipamientos urbanos ausentes; invasión de terrenos y áreas naturales; pérdida de identidad e integración social; paisajes urbanos poco alentadores; etc. Sin embargo, se suele confundir este tipo de expansión de concentrarse con la expansión urbana territorial en general. Lo cierto que es que existen varios tipos de expansión territorial de la ciudad. Hasta podríamos considerar que la densificación es una forma de expansión urbana que puede adoptar diversas estrategias una de ellas la vertical, especialmente cuando no hay el pacto con el territorio en la horizontalidad." (Chavoya et al., 2009).

En todo caso la arquitectura es: "el arte de proyectar y construir edificios". (REA, 2017a) Es decir: "Dar forma a los lugares donde vive la gente, no es más complicado que eso, pero tampoco más sencillo que eso". (Aravena, 2016). Como quien define: "La arquitectura es el arte y la ciencia de asegurarnos de que nuestras ciudades y edificios encajen realmente con la forma en que queremos vivir nuestras vidas: el proceso de manifestar nuestra sociedad en nuestro mundo físico". (Bjarke Ingels, 2017). La Lengua Española se define al urbanismo como "Conjunto de conocimientos relacionados con la planificación y desarrollo de las ciudades" definición a la que se agregan las ideas de Jenneret (1989) quien ve el urbanismo como el ordenamiento de los lugares que deben abrigar el desarrollo de la vida en las aglomeraciones urbanas y rurales, velando por las funciones básicas como el habitar, trabajar, recrearse y desplazarse. (Ducci, 2011) quien define el urbanismo como "el estudio y planeación de las 


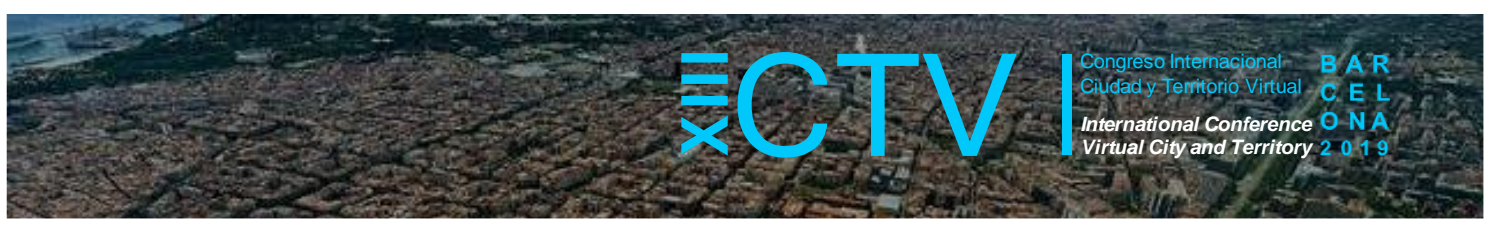

ciudades y de las regiones donde estas se asientan". (Grassi, 1959) quien toma el urbanismo como una ciencia, que se encarga de planificar y dar forma al territorio y la ciudad, buscando esculpir la estructura orgánica da la vida social y económica.

(Chavoya et al., 2009) el modelo de ciudad compacta, entendido como un espacio urbano que implica edificios verticales, es un entorno favorable para fortalecer o recrear cierta urbanidad e interacción social. A diferencia de la vivienda establecida a partir de casas individuales horizontales, generalmente en condominios cerrados, y de la cual se suele salir y entrar en automóvil individual sin ni siquiera pisar el espacio público entre la casa y el centro comercial, una ciudad densa favorece el tránsito de más población en el espacio público. La redensificación al proceso que busca el aumento de la densidad en zonas urbanas ya existentes. La densificación al proceso que busca el aumento de la densidad humana, entendida ésta como el número de población por unidad espacial. En este sentido: (REA, 2017b) define la vivienda como un lugar cerrado y cubierto construido para ser habito por personas.

\section{Localización y área de estudio}

Figura 1. Ubicación de Puerto Vallarta, Jalisco, México

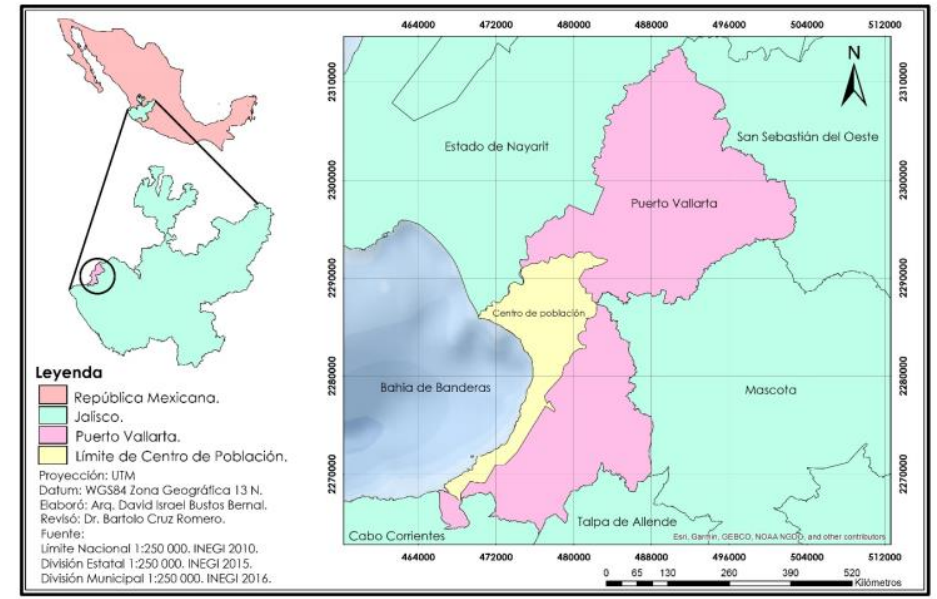

Fuente: Elaboración propia.

Figura 2. Objeto de estudio

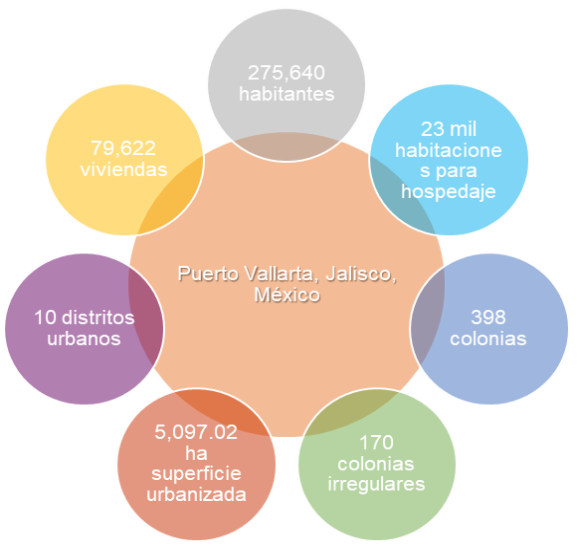

Fuente: (INEGI, 2015). 


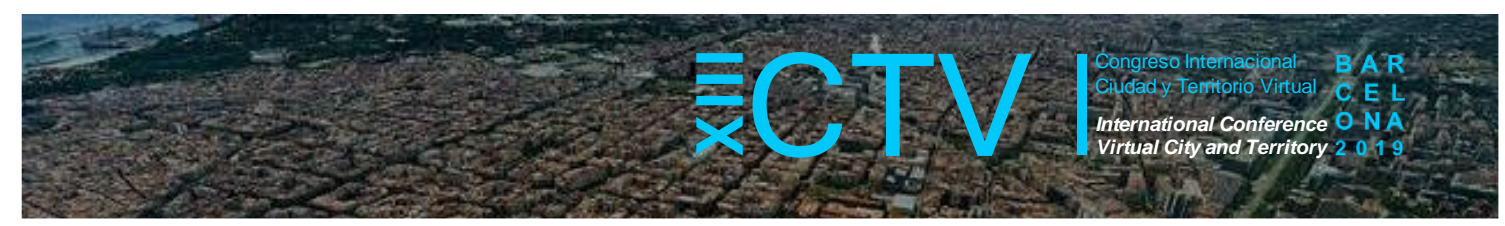

Figura 3. Marco teórico-conceptual

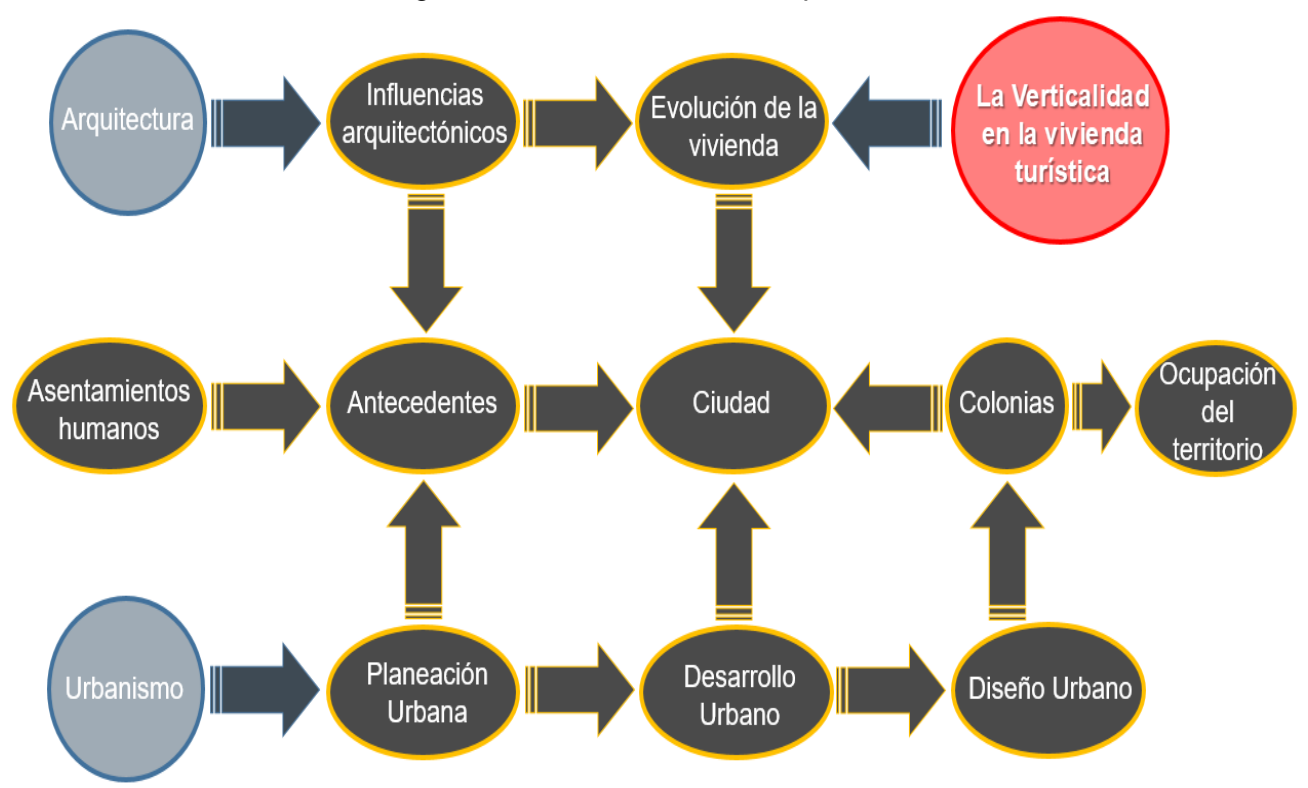

Fuente: Elaboración propia.

\section{Antecedentes}

Evolución histórica y desarrollo urbano de Puerto Vallarta, Jalisco, México.

\subsection{Etapa rural o urbanización temprana}

Tabla 1. Etapa rural o urbanización temprana

1851 Fundación de las Peñas de Santa María de Guadalupe

1857 Concesión de tierras a la empresa Unión en Cuale

1886 Elevación de Las Peñas de Santa María de Guadalupe a Comisaría política y judicial del municipio de Talpa de Allende

1888 Anexo al municipio de Sebastián del Oeste de la Comisaría de Las Peñas

1900 Se censa por primera vez la Comisaria de las Peñas

Fuente: (Baños Francia J, 2010; Cárdenas-Gómez \& Rodríguez-Bautista, 2012; Duarte, 2017)

\subsection{Elevación a municipio, etapa ejidal}

\section{Tabla 2. Elevación a municipio, etapa ejidal}

\begin{tabular}{|c|c|}
\hline 1918 & Elevación de Comisaría de Las Peñas a municipio y cambio de nombre a Puerto Vallarta. \\
\hline 1920 & Se integra la Comisión Local Agraria, se constituye el Ejido de Puerto Vallarta y el Fundo legal. \\
\hline 1930 & Se inaugura el primer generador eléctrico para dotar a la población de energía eléctrica. \\
\hline 1944 & Se inaugura la brecha Compostela-Puerto Vallarta. \\
\hline 1952 & Se pone en marcha el Programa de Progreso Marítimo (Marcha al mar) \\
\hline 1953 & $\begin{array}{l}\text { Se crea la Comisión de Planeación de la Costa de Jalisco como apoyo al Programa Marcha al Mar } \\
\text { del Presidente Adolfo Ruiz Cortines. }\end{array}$ \\
\hline 1954 & Inicia operaciones Mexicana de Aviación. \\
\hline 1956 & $\begin{array}{l}\text { Se comienzan a consolidar las colonias Emiliano Zapata y } 5 \text { de diciembre, al sur y al norte del } \\
\text { Fundo legal respectivamente. }\end{array}$ \\
\hline 1958 & $\begin{array}{l}\text { Se concluye la Central Eléctrica que dotaría a toda la población de energía eléctrica de manera } \\
\text { regular. }\end{array}$ \\
\hline 1958 & e \\
\hline
\end{tabular}




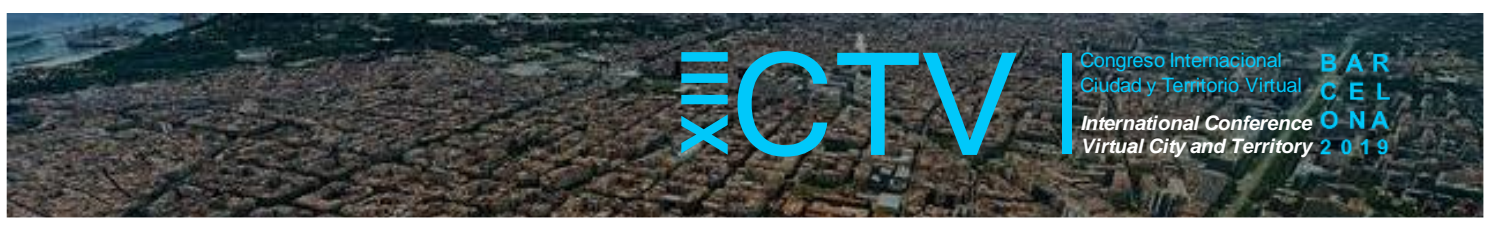

1963 Filmación de la película La Noche de la Iguana.

1965 Inicia el Gobierno de Francisco Medina Ascencio.

Fuente: (Baños Francia J, 2010; Cárdenas-Gómez \& Rodríguez-Bautista, 2012)

\subsection{Auge urbano y regularización de la tenencia de la tierra}

\section{Tabla 3. Auge urbano y regularización de la tenencia de la tierra}

1968 El 31 de mayo de 1968 el poblado de Puerto Vallarta es elevado a la categoría de ciudad por el H. Congreso del estado de Jalisco.

1970 Aparecen las colonias Lázaro Cárdenas, López Mateos, Valentín Gómez Farías, Versalles y Olímpica.

1970 Se crea el Fideicomiso Traslativo de Dominio Bahía de Banderas.

1972 Comienzan a aparecer edificaciones en la que hoy es la colonia Versalles.

1973 Se divide el Fideicomiso Traslativo de Dominio Bahía de Banderas y se crea el Fideicomiso Traslativo de Dominio Puerto Vallarta.

1975 Se elabora el Plan General Urbano de Puerto Vallarta por la Junta General de Planeación y Urbanización de Jalisco.

1976 Se construyen una gran cantidad de hoteles.

1982 Se devalúa el peso, situación que contribuyó a que el destino turístico se volviera más atractivo para los visitantes extranjeros.

1984 Se edifica Vallarta 500 por parte de la iniciativa privada.

1985 Se edifica Vallarta 750 por parte de la iniciativa privada y el fraccionamiento Tamarindos en Ixtapa de inversión Gubernamental.

1985 Comienzan a aparecer asentamientos humanos irregulares en los predios Ejidales aledaños a El Pitillal.

1985 Aparecen las colonias Villa Las Flores, Aramara, Barrio Santa María, Niños héroes, Las Gaviotas, El Caloso y El Remance.

1988 Se incorporan las colonias $1^{\circ}$ de Mayo, Ramblases, Agua Azul, Alta Vista, Amapas, La Bobadilla, El Coapinole, Lomas del Coapinole, Villa de Guadalupe, Del Toro, Palmar de Aramara y la Aurora y se consolida Mismaloya y Boca de Tomatlán

1993 Aparecen las colonias Mojoneras, La Educación, San Esteban, Morelos y Pavón, Lomas del Calvario, Fovisste 100, Infonavit, Las Aralias, Los Sauces y Cristóbal Colón.

1993 Se concluye la edificación de Marina Vallarta y con ella la construcción de una gran cantidad de hoteles.

Fuente: (Baños Francia J, 2010; INEGI, 2015)

\subsection{Etapa de urbanización masiva o planeación urbana local}

Con la reforma al artículo 27 Constitucional de 1992 y la nueva Ley Agraria, se permitió el cambio de estatus de la tierra de propiedad colectiva comunal a privada, ocasionando el aumento en la urbanización y la venta masiva de las parcelas ejidales promovida por un gran número de inversionistas privados; situación que llevó a un acelerado crecimiento urbano, que superó la demanda de suelo habitable. En 1997 se aprobó el Plan de Desarrollo Urbano de Centro de Población como una herramienta de Planeación del crecimiento urbano de la ciudad, constituyéndose como la primera herramienta emanada por el propio municipio. (Duarte, 2017) En 2006 se autoriza la publicación de un nuevo Plan de Desarrollo Urbano de Centro de Población, mismo que fue suspendido, según fuentes de la Dirección de Planeación Urbana del Municipio, por una queja interpuesta por un sector de la población. Sin embargo, alrededor del año 2012, un Juez dictaminó que dicho Plan de Desarrollo Urbano del Centro de población 2006, era legal, por lo que la suspensión quedó revocada, pero ya era anacrónico y de difícil aplicación relativamente. 


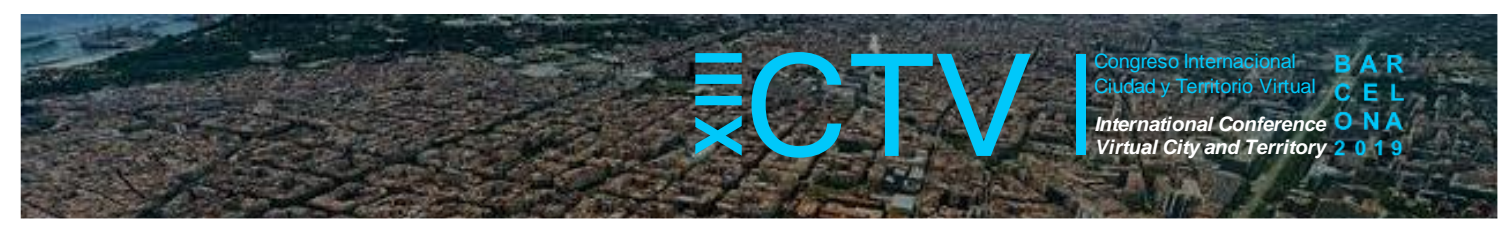

El 19 de agosto de 2011, a través del acuerdo de Ayuntamiento № 0571/2011, el municipio fue dividido formalmente en 10 distritos urbanos, delimitados por el centro de población. A partir de esta distritación, el Plan de Desarrollo Urbano del Centro de Población 2006, comenzó a actualizarse de forma parcial en cada uno de sus 10 Distritos urbanos (Duarte, 2017), pero resultó insuficiente.

A partir del año 2012 se actualizó una gran cantidad de los nuevos distritos urbanos, además de la creación del Plan Parcial de Desarrollo Urbano Arroyo Quelitán y el Plan Parcial de Desarrollo Urbano Las Palmas con el propósito de ordenar la gran cantidad de asentamientos humanos irregulares que comenzaban a aparecer y consolidarse en esta zona.

Dichas actualizaciones se enlistan a continuación:

- EL 2 de mayo de 2012, como actualización del Plan de Desarrollo Urbano de Centro de Población 2006, se publica en la Gaceta Municipal Puerto Vallarta con el número de Acuerdo de Ayuntamiento 0785/2012 el Plan Parcial de Desarrollo Urbano del Distrito Urbano 2.

- EL 9 de mayo de 2012, como actualización del Plan de Desarrollo Urbano de Centro de Población 2006, se publica en la Gaceta Municipal Puerto Vallarta con el número de Acuerdo de Ayuntamiento 0808/2012 el Plan Parcial de Desarrollo Urbano del Arroyo Quelitán.

- EL 17 de septiembre de 2012, como, actualización del Plan de Desarrollo Urbano de Centro de Población 2006 se publica en la Gaceta Municipal Puerto Vallarta con el número de Acuerdo de Ayuntamiento 0930/2012 el Plan Parcial de Desarrollo Urbano del Distrito Urbano 9.

- EL 18 de septiembre de 2012, como actualización del Plan de Desarrollo Urbano de Centro de Población 2006, se publica en la Gaceta Municipal Puerto Vallarta con el número de Acuerdo de Ayuntamiento 0929/2012 el Plan Parcial de Desarrollo Urbano del Distrito Urbano 6.

- El 18 de septiembre de 2012, como actualización del Plan de Desarrollo Urbano de Centro de Población 2006, se publica en la Gaceta Municipal Puerto Vallarta con número de Acuerdo de Ayuntamiento 0928/2012 el Plan Parcial de Desarrollo Urbano del Sub distrito Urbano 5B "Estero El Salado".

- EL 18 de septiembre de 2012, como actualización del Plan de Desarrollo Urbano de Centro de Población 2006, se publica en la Gaceta Municipal Puerto Vallarta con el número de Acuerdo de Ayuntamiento 0926/2012 el Plan Parcial de Desarrollo Urbano del Distrito Urbano 3.

- EL 27 de septiembre de 2012, como actualización del Plan de Desarrollo Urbano de Centro de Población 2006, se publica en la Gaceta Municipal Puerto Vallarta con el número de Acuerdo de Ayuntamiento 0927/2012 el Plan Parcial de Desarrollo Urbano del Distrito Urbano 5-A.

- EL 18 de enero de 2015, como actualización del Plan de Desarrollo Urbano de Centro de Población 2006, se publica en la Gaceta Municipal Puerto Vallarta con número de Acuerdo de Ayuntamiento 065/2015 el Plan Parcial de Desarrollo Urbano del Distrito Urbano 4.

- EL 18 de enero de 2015, como actualización del Plan de Desarrollo Urbano de Centro de Población 2006, se publica en la Gaceta Municipal Puerto Vallarta con número de Acuerdo de Ayuntamiento 066/2015 el Plan Parcial de Desarrollo Urbano del Distrito Urbano 10.

Esto muestra la enorme presión demográfica y ecológica, cuya respuesta los planes urbanos intentan resolver, pero al cabo de un tiempo se han ido desbordando. 


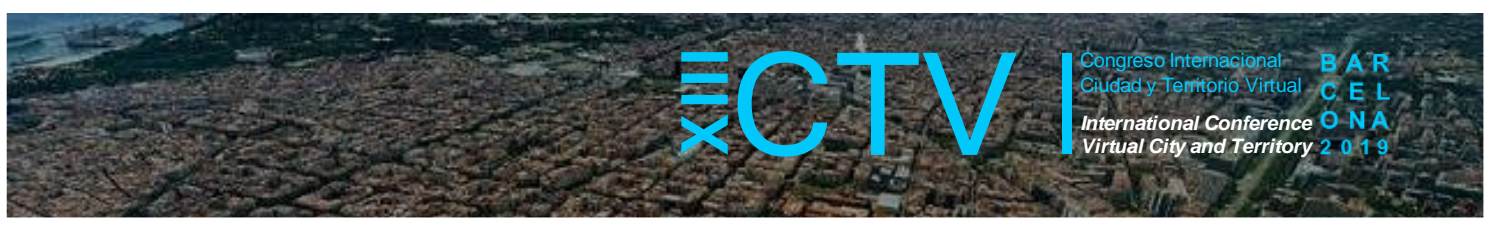

\subsection{Atributos arquitectónicos}

(Baños Francia J, 2010; Chavoya et al., 2009). La fisionomía original de la arquitectura de Puerto Vallarta, proviene de las construcciones serranas. Con el paso del tiempo, los avances de las tecnologías, favorecieron la introducción de nuevas manifestaciones. Actualmente, dos imaginarios: conservar la tradición arquitectónica e innovar el ejercicio del diseño.

\section{Figura 4. Arquitectura Serrana (1851-1948)}

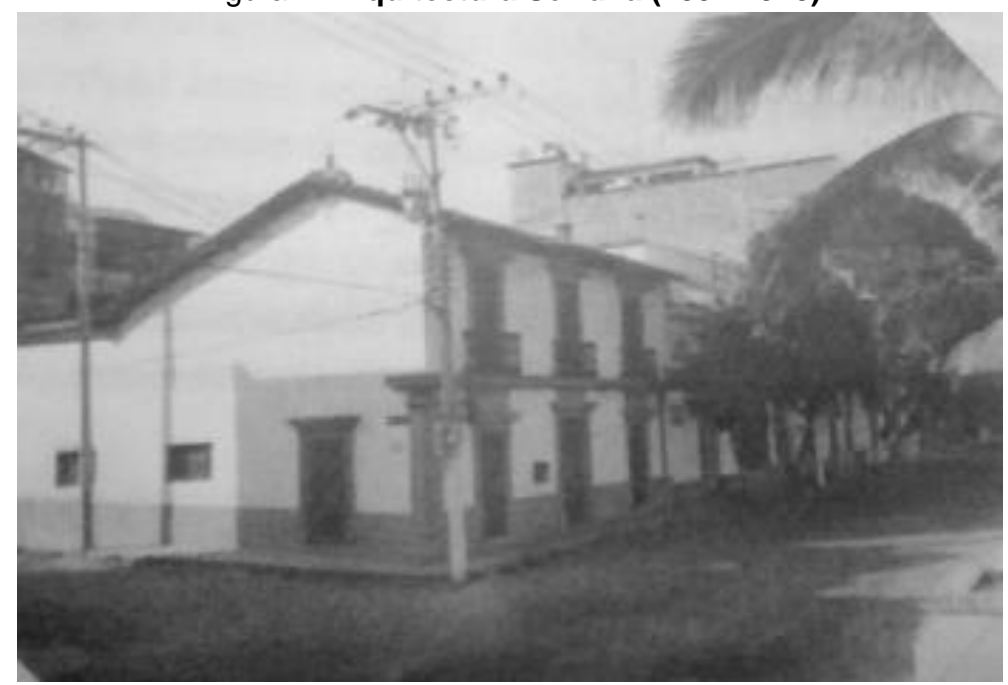

Fuente: Dominio Público

Características, según (Baños Francia J, 2010; Chavoya et al., 2009): Aire rústico y pueblerino, la traza urbana se conforma con calles que se entrecruzan, la traza se aplica tanto en lo plano como en las montañas, las calles "suben y bajan", las calles y andadores se pavimentan con materiales de la región (piedra bola), empleo predominante de materiales de la región. Los muros son de adobe, las cubiertas se hacen con vigas, polines, morillos y fajillas, muros enjarrados sobre puertas y ventanas, 3 colores predominantes, gris, blanco y rojo, la teja es de barro cocido y la altura es proporcional a lo ancho de las calles.

Figura 5. Arquitectura Funcionalista (1948-1960)

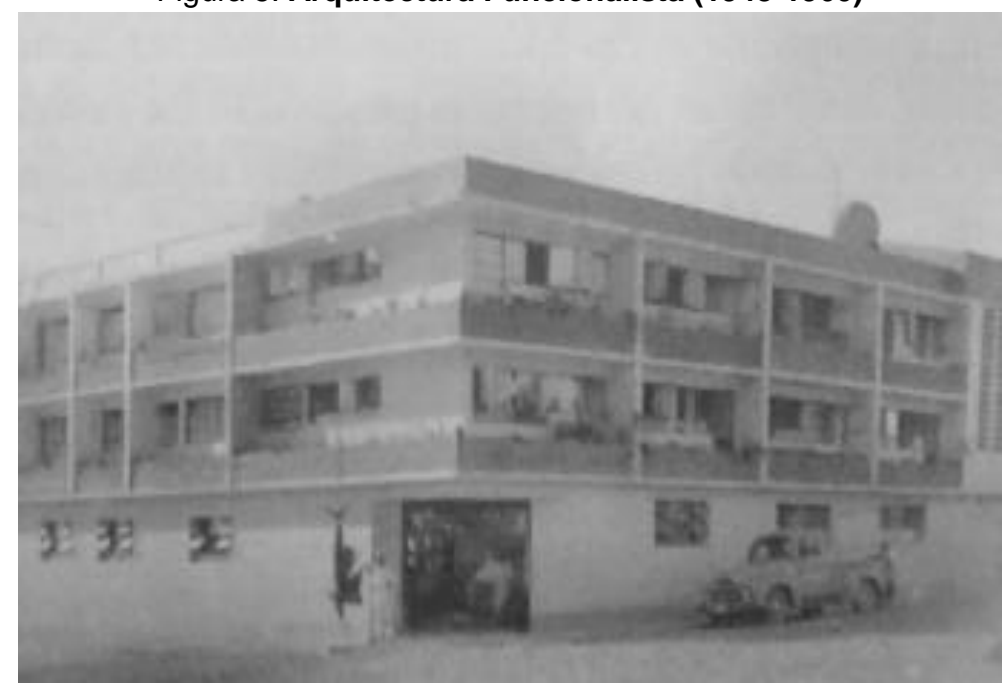

Fuente: Dominio Público. 


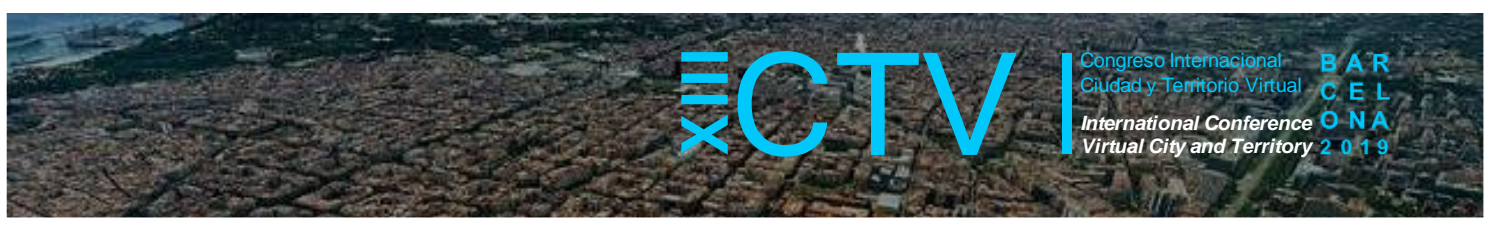

Características (Baños Francia, 2010; Chavoya et al., 2009): Los muros se construyen con ladrillo cocido, reforzados con castillos y través de concreto, las puertas y ventanas se disponen de forma horizontal, carente de moldura, la cancelería es de herrería y cristal, se presentan balcones volados a 90 centímetros, las cubiertas son planas y se instalan bajantes para las aguas pluviales y la incrementación del número de niveles en las fincas, presentándose de 3 a 5 pisos en promedio.

\section{Figura 6. Arquitectura Vallarta (1950-1970)}

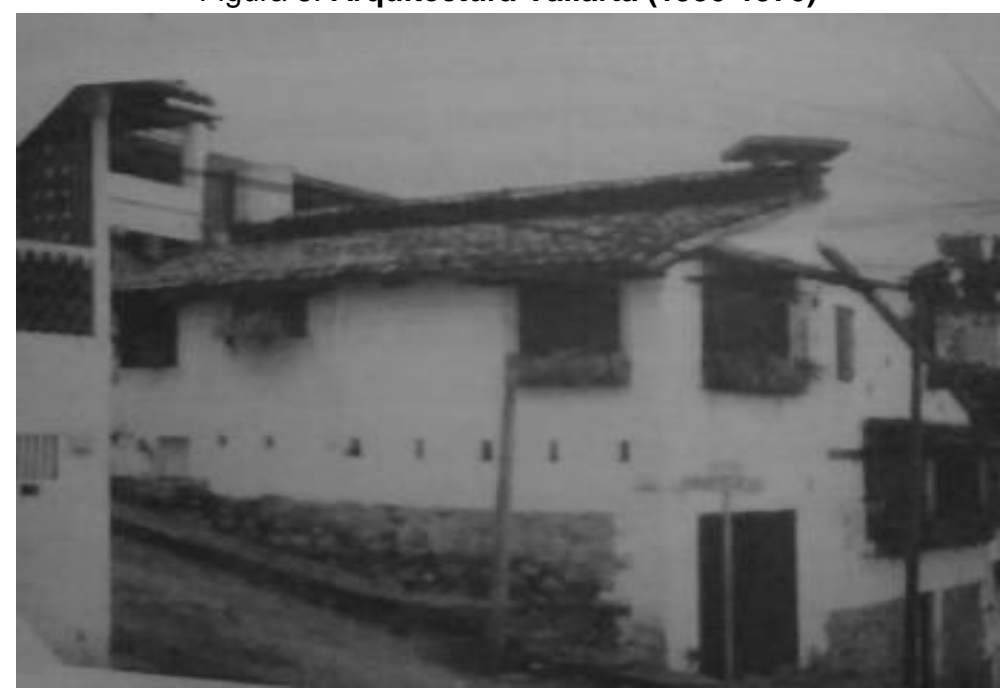

Fuente: Dominio Público.

Características (Baños Francia, 2010; Chavoya et al., 2009): Recuperación de la arquitectura local, de pueblo serrano, se incorporaron materiales y sistemas constructivos contemporáneos, en una mezcla de tradición y modernidad, se destaca la obra de Freddy Romero Escalante quien no era arquitecto de profesión pero tenía una sensibilidad artística muy refinada, sobresalió, los equipales, las cocinas con sus pretiles de azulejos, los arcos de ladrillos, las ventanas con de persianas de madera, las celosías y las jardineras, aparece la cúpula arcos de medio punto y punto buscado.

Figura 7. Arquitectura para el turismo (1970-1990)

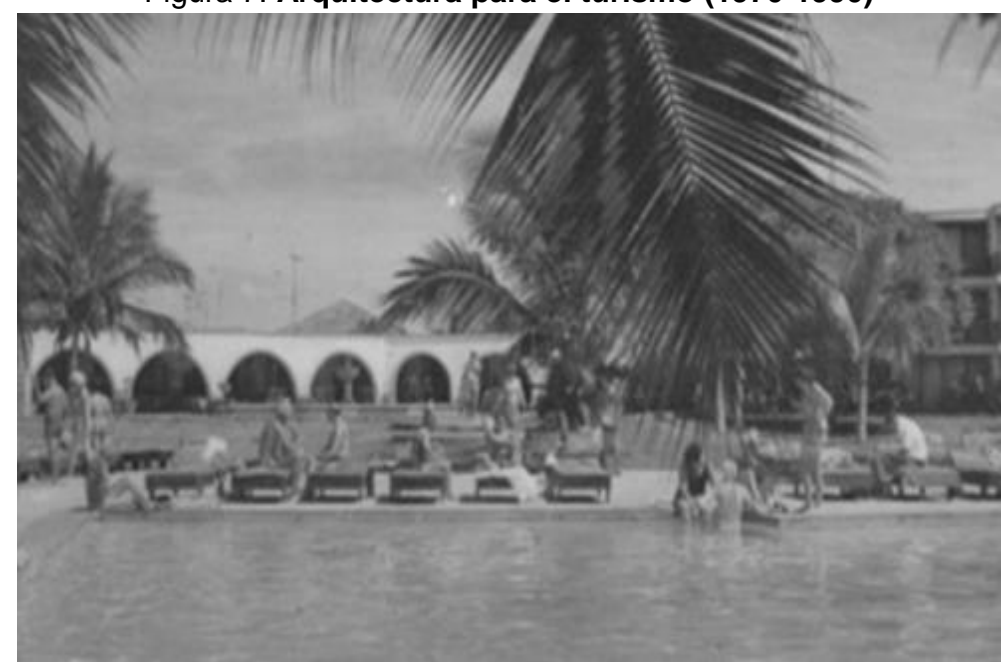

Fuente: Dominio Público. 


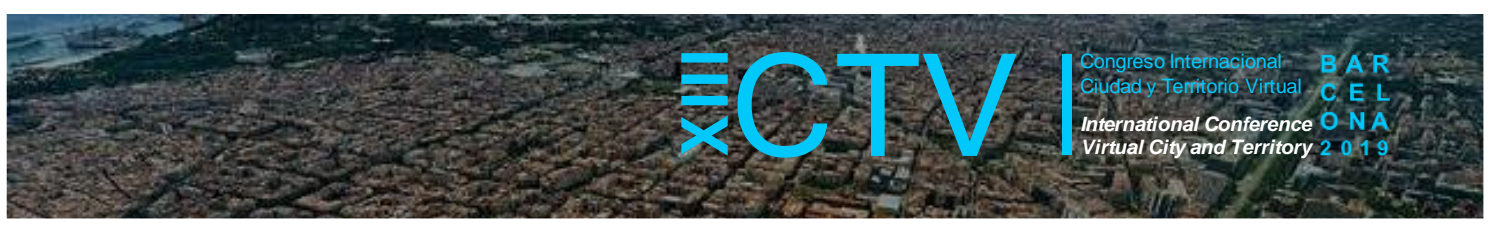

Características (Baños Francia J, 2010; Chavoya et al., 2009): Utilización de elementos formales, tomados de la arquitectura serrana tradicional y del estilo Vallarta, pero incorporados de una escala diferente, ya no doméstica sino de grandes proporciones, utilizando principalmente en la construcción de hoteles, se adapta los nuevos usos funcionales y simbólicos, para la transmisión la sensación de estar en un "pueblito tradicional". Se emplean las cúpulas, arcos de ladrillo aparente y cubiertas de teja de barrio tradicional y la altura de los edificios era de la altura de las palmeras.

Figura 8. Arquitectura Contemporánea (1990-2019)
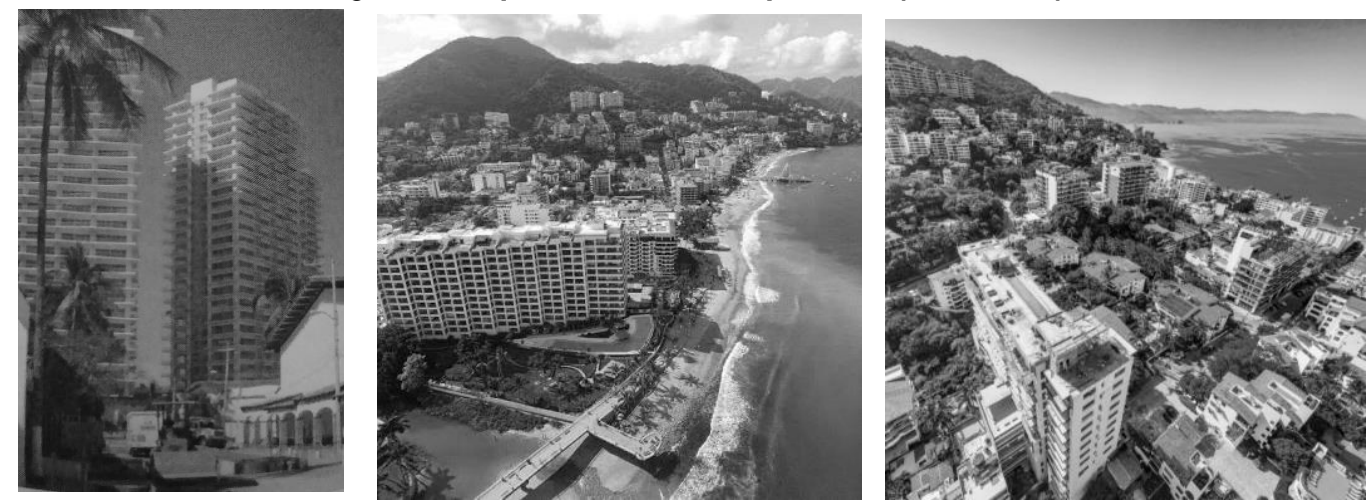

Fuente: Dominio Público; 3d.casa/360/soho

Características (Baños Francia J, 2010; Chavoya et al., 2009): Los edificios se diseñan se diseñan con el objetivo de asegurar la recuperación económica de los inversionistas y no como una aportación al tejido arquitectónico local y la violación contaste a la normatividad urbana, la edificación de gran altura, la afectación al medio ambiente y alteración al paisaje natural y arquitectónico.

\subsection{Ensanches urbanos}

(Cárdenas-Gómez \& Rodríguez-Bautista, 2012) Los ensanchamientos urbanos de Puerto Vallarta, Jalisco, durante un siglo (xx); se analiza la traza urbana y los cambios que ha vivido este municipio jalisciense a partir de relacionar el crecimiento urbano. El periodo de estudio abarca de 1913 a 2000.

Figura 9. Puerto las Peñas (1913)

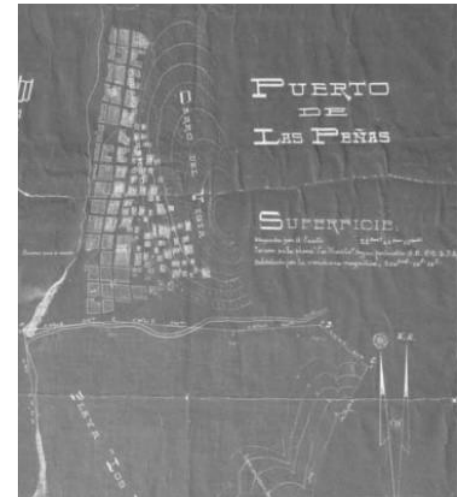

Fuente: Gobierno de Jalisco. Archivo Histórico del estado de Jalisco. Número de clasificación PI211913222; (CárdenasGómez \& Rodríguez-Bautista, 2012). 


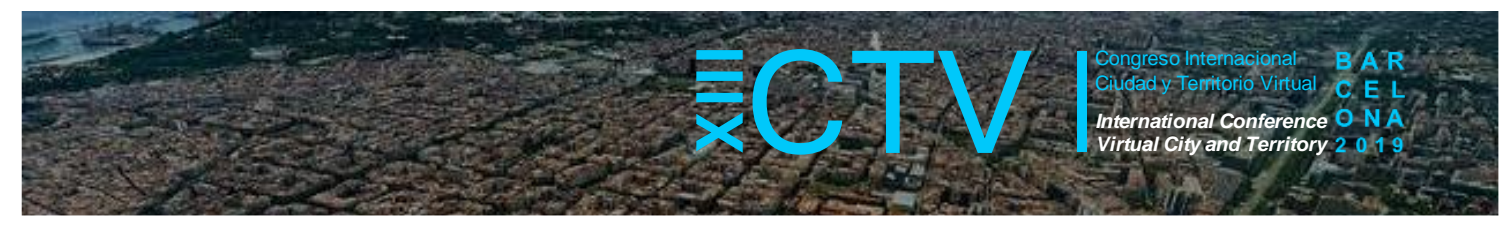

Características: Contaba con 90 manzanas, 4,800 habitantes y 485 casas.

Figura 10. Puerto Vallarta (1920)

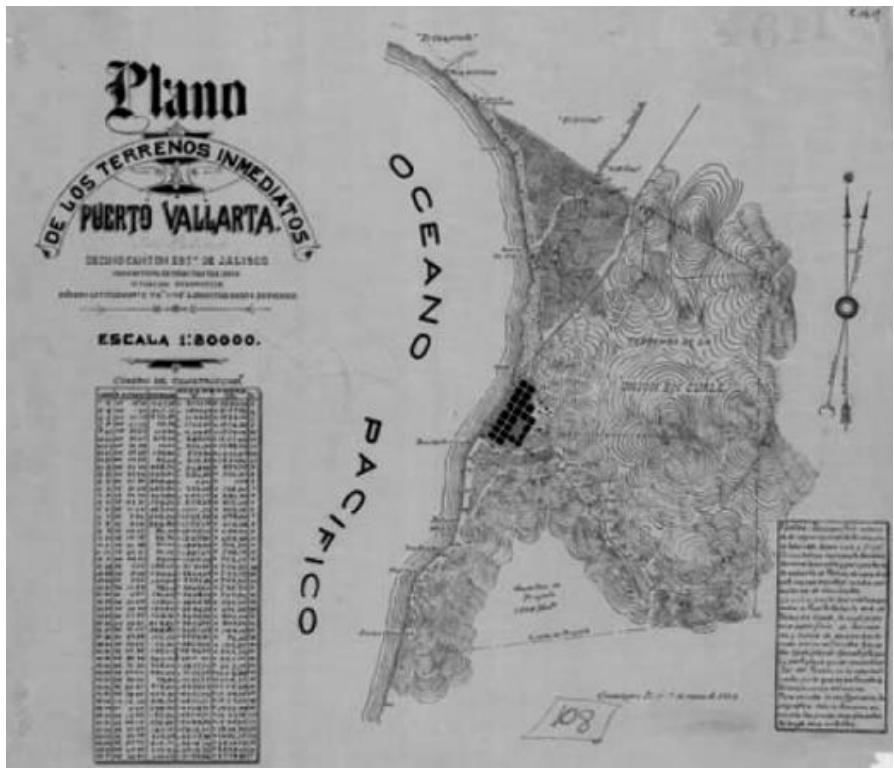

Fuente: Universidad de Guadalajara (2012), número de clasificación 990, Jalisco.; (Cárdenas-Gómez \& RodríguezBautista, 2012).

Características: Contaba con 24 manzanas y 2,606 habitantes.

Figura 11. Puerto Vallarta (1940-1943)

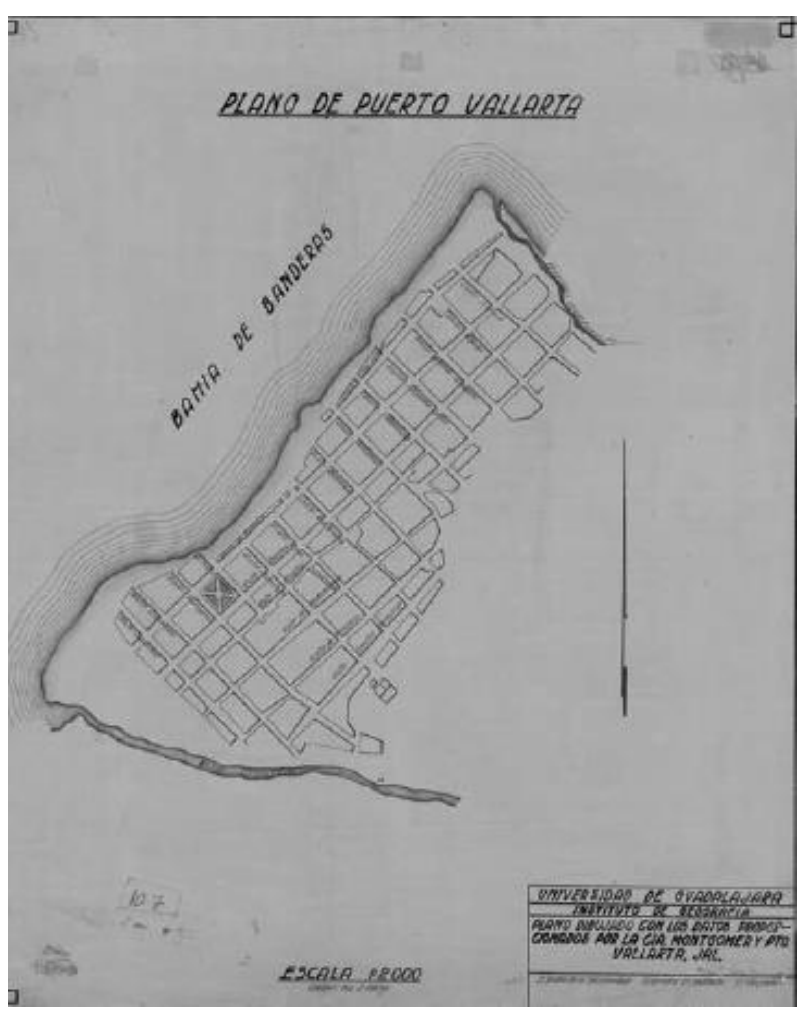

Fuente: Universidad de Guadalajara (2012), número de clasificación 1179, Jalisco.; (Cárdenas-Gómez \& RodríguezBautista, 2012). 


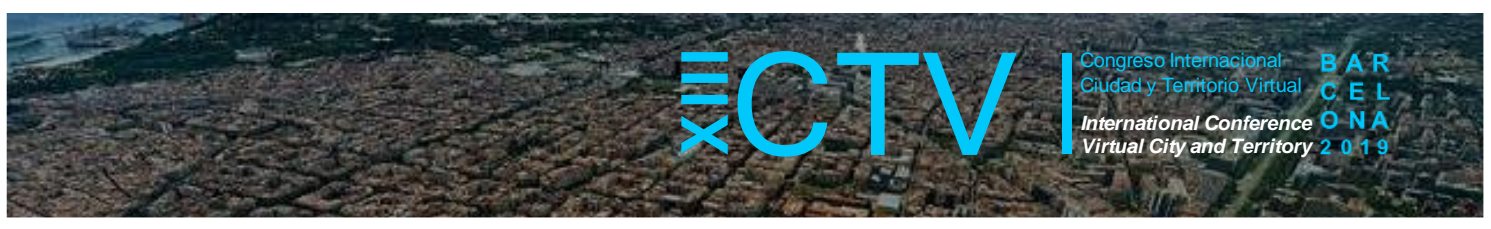

Características: Contaba con 86 manzanas y 10,471 habitantes y en el año1950, contaba con 10,801 habitantes y el trazo de la colonia Emiliano Zapata y 5 de diciembre.

Figura 12. Puerto Vallarta (1973-1990)

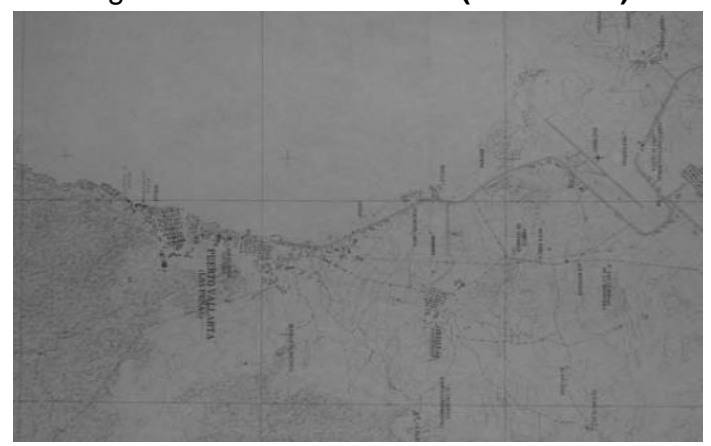

Fuente: INEGI, 1973; (Cárdenas-Gómez \& Rodríguez-Bautista, 2012).

Características: Conmemoración del centenario de la fundación de Puerto Vallarta (1951), filmación de la película La Noche de la Iguana (1963). Francisco Medina Ascencio eleva a Puerto Vallarta a rango de ciudad (1968), reunión de los presidentes Richard Nixon y Gustavo Díaz Ordaz (1970) y se crea el Fideicomiso Bahía de Banderas, con el objeto de regularizar la tierra y dar certidumbre a los inversionistas (1970).

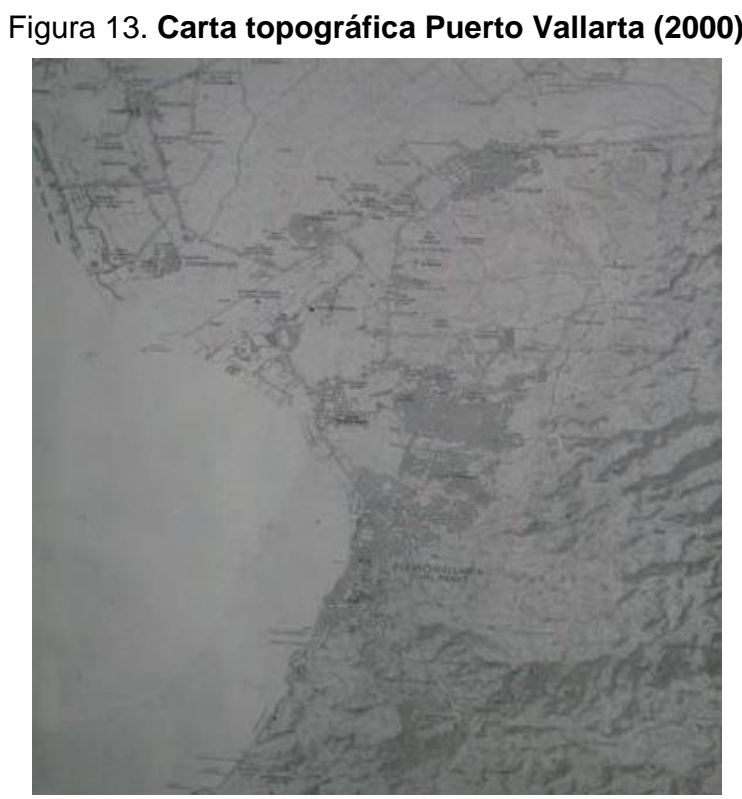

Fuente: INEGI, 2000; (Cárdenas-Gómez \& Rodríguez-Bautista, 2012).

Características: Había 130 manzanas que componen la cabecera municipal y 184 728habitantes.

Vallarta ha sido y sigue siendo un caos urbano que muestra dos caras distintas: una, la zona turística compuesta por el área centro del poblado, La Marina Vallarta y la franja hotelera (la sur y norte); y la otra, las colonias populares. (Gómez, 2010: 21 


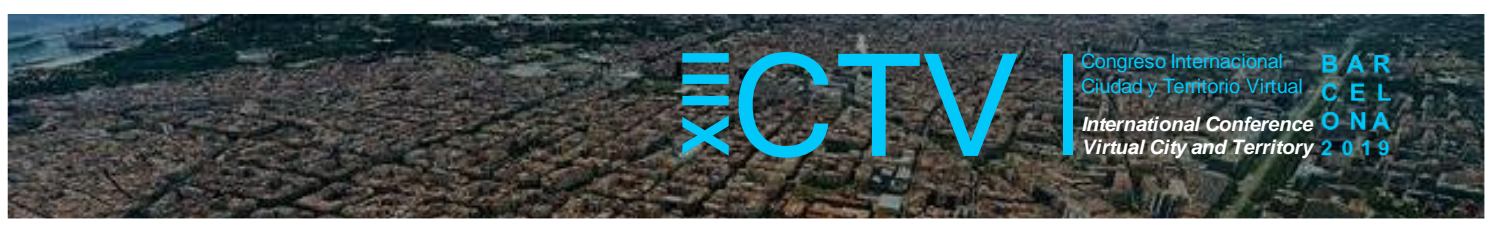

\subsection{Población del municipio}

Tabla 4. Crecimiento poblacional

\begin{tabular}{|c|c|}
\hline Censo & Total de habitantes \\
\hline 1921 & 2218 \\
\hline 1930 & 10245 \\
\hline 1940 & 10471 \\
\hline 1950 & 10801 \\
\hline 1960 & 15462 \\
\hline 1970 & 35911 \\
\hline 1990 & 57911 \\
\hline 2000 & 111457 \\
\hline 2010 & 184728 \\
\hline 2015 & 255681 \\
\hline
\end{tabular}

Fuente: Censos Generales de Población 1921, 1930, 1940, 1950, 1960, 1970, 1980, 1990, 2000, 2010 y 2015.

\subsection{Planeación urbana en la actualidad}

Con el explosivo crecimiento urbano de Puerto Vallarta a partir de la década de 1970, se hizo evidente la necesidad de instrumentar mecanismos para potenciar las bondades del crecimiento y reducir los impactos negativos de la expansión local. Los instrumentos de planeación urbana en Puerto Vallarta más destacados son: Plan de Ordenamiento Urbano de la desembocada del Rio Ameca (1973), Plan de Desarrollo Urbano de Centro de Población (1975), Plan General Urbano Puerto Vallarta (1982), Proyecto de actualización del Plan General Urbano (1989), Plan Parcial de Urbanización y control de la Edificación Ingreso Norte, Plan de desarrollo urbano de centro de población de Puerto Vallarta, 1997 y Plan de Desarrollo Urbano de Centro de Población 2006. El 19 de agosto de 2011, a través del acuerdo de Ayuntamiento $\mathrm{N}^{\circ}$ 0571/2011, el municipio fue dividido formalmente en 10 distritos urbanos, delimitados por el centro de población.

Figura 14. Distritación del límite del centro de población

Fuente: Planeación Urbana, 2013.

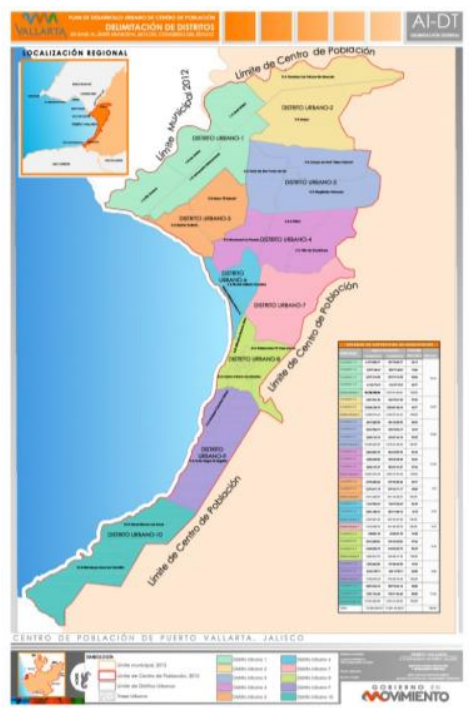




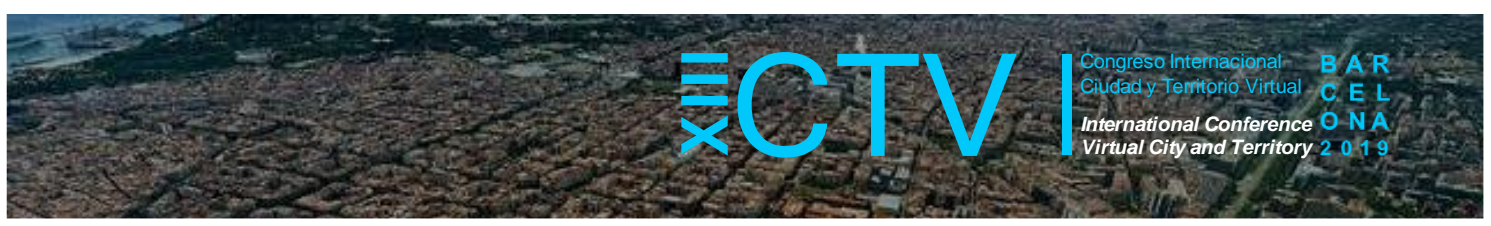

\section{Resultados}

A través del método analítico de tipo cualitativo, mediante la técnica de la observación participante en los lugares de interés previamente seleccionados se observa que en los últimos años Puerto Vallarta ha aumentado hasta el año del 2015 con una población de 275640 habitantes según datos de censos de población (INEGI, 2015), debido a sus oportunidades de desarrollo y quienes han decido habitar y quedarse en el ámbito comercial. El turismo es la principal fuente de ingreso, pero es por eso que en diferentes sectores "colonias" de la ciudad presenta un crecimiento acelerado crece caótica y mal ordenada o simplemente; edificación de construcciones mal planeadas para el crecimiento de la ciudad.

\section{Conclusiones}

La ciudad de Puerto Vallarta, Jalisco, experimenta nuevos procesos de transformación urbana en términos de ocupación del suelo y de redensificación de áreas consolidadas promovidas por el sector inmobiliario. En estos nuevos esquemas la vivienda turística juega un papel determinante en la reconfiguración territorial de la ciudad, toda vez que detona la tendencia a la verticalización promoviendo a su vez nuevos atributos arquitectónicos con la modificación del paisaje urbano, todo esto inserto hasta el momento en un modelo extractivo. El turismo ha sido el factor el cual ha influido en el crecimiento de la ciudad de Puerto Vallarta, el más importante en el sector inmobiliario quien ha tomado posicionamiento en el hábitat de la población, en este caso más a extranjeros, quienes han elegido a Puerto Vallarta como un destino para vivir, por ser una ciudad tranquila y con atribuciones específicas, por tal motivo deciden radicar o comprar una propiedad o bien común en la ciudad. Hay algunos efectos positivos en ello, pero en general el rápido crecimiento demográfico. La consolidación turística de la ciudad de Puerto Vallarta, Jalisco, ha generado flujos migratorios y de capital financiero, propiciando esquemas de crecimiento urbano en algunas áreas del municipio, dando lugar a un proceso direccionado a la verticalidad en la vivienda turística, exigiendo nuevos atributos arquitectónicos y actuaciones inéditas en la planeación urbana de la ciudad.

Figura 9. Paisaje Urbano actual de Puerto Vallarta, Jalisco, México.

Fuente: 3d.casa/360/soho

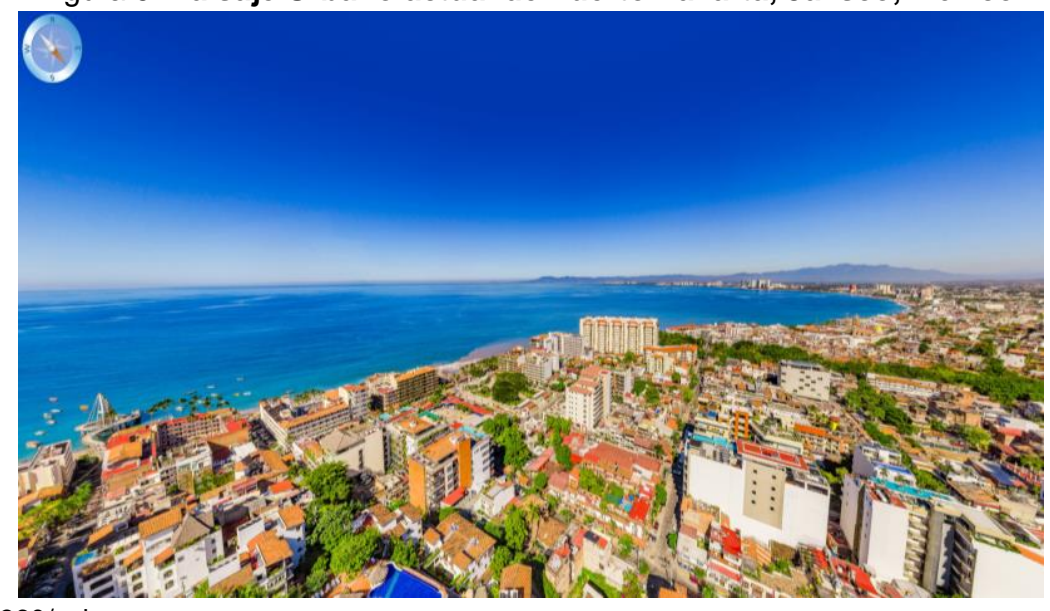




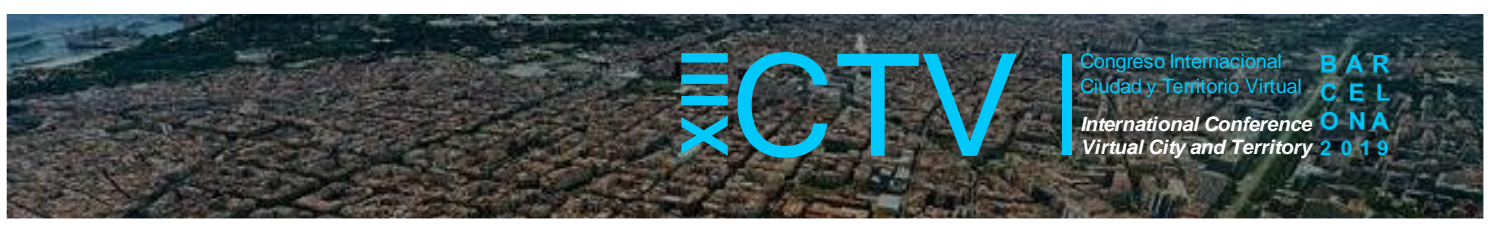

Agradecimientos: Gracias a todas las personas que tanto directa como indirectamente contribuyeron a la realización de esta investigación que aún está en proceso. No es un camino fácil, pero está lleno de experiencias buenas y malas y gracias a su apoyo, su bondad, su confianza y amor, esto que parecía complejo fue algo más bien agradable y provechoso. A CONACYT, por el apoyo económico, a la Universidad de Guadalajara, Centro Universitario de la Costa, al Posgrado Maestría en Ciencias para el Desarrollo, la sustentabilidad y el Turismo por su apoyo incondicional en cada momento. Les agradezco a todos ustedes y hago presente el gran cariño y afecto que les tengo y a quienes han estado momento a memento en esta etapa de investigación.

Contribuciones de los autores: Éste articulo ha sido escrito por el primer autor, con la dirección y contribución del segundo autor.

Conflicto de Intereses: Los autores declaran que no hay conflicto de intereses.

\section{Bibliografía}

Aravena, A. (2016). Definión de Arquitectura.

Baños Francia, J. A. (2010). Arquitectura y urbanismo en Puerto Vallarta. Una mirada a la construcción de una ciudad turistica de litoral. Astra Ediciones SA de CV.

Bjarke Ingels. (2017). Definición de Arquitectura.

Cárdenas-Gómez, E., \& Rodríguez-Bautista, J. (2012). La transformación urbana de Puerto Vallarta, Jalisco. Espacios Públicos, 15(34), 208-230. Retrieved from http://www.redalyc.org/articulo.oa?id=67623463016\%0Ahttp://www.redalyc.org/pdf/676/676234 63016.pdf

Chavoya, J. I., García, J., \& Rendón, H. J. (2009). Una reflexión sobre el modelo urbano: ciudad dispersa-ciudad compacta. 5Th International Conference Virtual City and Territory, 3750.

Duarte, J. R. S. (2017). ASENTAMIENTOS HUMANOS IRREGULARES EN EL MUNICIPIO DE PUERTO VALLARTA, JALISCO, MÉXICO.

Ducci, M. E. (2011). Conceptos Básicos de urbanismo. El Urbanismo Y La Planificación, 21,36.

Grassi, F. F. (1959). Urbanismo para todos.

INEGI. (2015). Prontuario de información geográfica municipal de los Estados Unidos Mexicanos. Yahualica de González Gallo, Jalisco. Clave geoestadística 14118.

REA. (2017a). Arquitectura. Retrieved from https://dle.rae.es/?id=3dyUvi4

REA. (2017b). Vivienda. Retrieved from https://dle.rae.es/?id=byF4Mc7 